ORIGINAL RESEARCH

\author{
M. Ribo \\ M. Rubiera \\ J. Pagola \\ D. Rodriguez-Luna \\ P. Meler \\ A. Flores \\ J. Alvarez-Sabin \\ C.A. Molina
}

\section{Bringing Forward Reperfusion with Oxygenated Blood Perfusion beyond Arterial Occlusion during Endovascular Procedures in Patients with Acute Ischemic Stroke}

BACKGROUND AND PURPOSE: High recanalization rates achieved with endovascular procedures are not always followed by the expected clinical improvement. These time-consuming procedures imply a delayed reperfusion despite the capacity of earlier intravascular microcatheter bypass to the ischemic tissue beyond the clot. We aimed to explore the safety and feasibility of MOB beyond the clot.

MATERIALS AND METHODS: We studied patients with stroke undergoing endovascular procedures. The timing of procedural steps was recorded. We then explored the safety and feasibility of repeated femoral artery $\mathrm{MOB}$ injections beyond the occlusion every time the clot was crossed in 17 patients. Pre- and postocclusion flow was continuously monitored with TCD.

RESULTS: We studied 60 patients (mean age, $70 \pm 11$ years; median NIHSS score, 20; IR, 18-21). Of them, $33(55 \%)$ received IV-tPA before the endovascular procedure. The following arteries were occluded: the MCA $(63.3 \%, n=38)$ and the ICA $(36.6 \%, n=22)$. The TSO to arterial puncture was $193 \pm 77$ minutes. The occluding clot was successfully crossed with the microcatheter in 46 patients (76.6\%; mean TSO, $228 \pm 82$ minutes). Recanalization was achieved in 44 patients (73.2\%; mean TSO, $328 \pm 144$ minutes). Repeated MOB injections were performed in 17 patients. Patients with/without $\mathrm{MOB}$ presented with similar baseline characteristics. The median number of $\mathrm{MOB}$ injections was 2 (IR, 2-3), and the median injected blood volume was $40 \mathrm{~mL}$ (IR, 27.5-50). The mean time from first $\mathrm{MOB}$ to arterial recanalization was $136 \pm 86$ minutes. During $\mathrm{MOB}$, a nonpulsatile flow appeared in previously nonvisible distal branches on TCD.

CoNCLUSIONS: In this small series, oxygenated blood delivered through a microcatheter positioned distal to the site of occlusion was feasible and safe. Until final recanalization is achieved, MOB injections may generate intermittent reperfusion for up to 2 hours.

\begin{abstract}
ABBREVIATIONS: $\mid \mathrm{A}=$ intra-arterial; $I \mathrm{ICA}=$ internal carotid artery; $I \mathrm{R}=$ interquartile range; $\mathrm{IV}=$ intravenous; $\mathrm{MCA}=$ middle cerebral artery; $\mathrm{MOB}=$ microcatheter oxygenated blood infusion; mRS = modified Rankin Scale; NIHSS = National Institutes of Health Stroke Scale; TCD = transcranial Doppler sonography; TIMI = Thrombolysis in Myocardial Infarction; tPA = tissue plasminogen activator; TSO $=$ time from symptom onset
\end{abstract}

R ecanalization, spontaneous ${ }^{1}$ or pharmacologically induced, ${ }^{2}$ of a major intracranial vessel after stroke is not infrequent; however, it usually occurs too late for tissue salvage. Final infarct extension and outcome strongly depend on the time of ischemia elapsed between initial vessel occlusion and recanalization. ${ }^{3}$ Approved therapies such as IV-tPA ${ }^{4,5}$ or direct catheter-mediated clot retrieval ${ }^{6-8}$ aim to advance recanalization in time and restore blood flow in the penumbra before irreversible damage occurs. Until blood flow is restored, "time is brain""; therefore, any measure that will reperfuse the endangered penumbral brain will potentially "buy time" until recanalization is achieved.

The high rates of arterial recanalization achieved with endovascular procedures are not always accompanied by the expected clinical improvement. ${ }^{8}$ During IA revascularization procedures,

Received March 28, 2010; accepted after revision May 20.

From the Unitat Neurovascular, Servei de Neurologia, Hospital Vall d'Hebron, Barcelona, Spain.

Marc Ribo is the recipient of a grant by the Instituto de Salud Carlos III (PS09/01660).

Please address correspondence to Marc Ribo, MD, Passeig Vall d'Hebron 119-129, Barcelona 08035, Spain; e-mail: marcriboj@hotmail.com

DOI 10.3174/ajnr.A2221 microcatheter access to the clot and to the distal vasculature beyond the occluding thrombus is usually achieved within minutes from procedure initiation. Unfortunately, the time invested to achieve final recanalization either with IA-tPA or mechanical clot retrievers is usually much longer; this time span implies a delayed reperfusion of the penumbral tissue.

Anecdotal cases suggested intermittent MOB beyond the occluding clot as a possible neuroprotective strategy. ${ }^{10} \mathrm{We}$ aimed to explore the safety and feasibility of repeated MOB beyond the occluding clot.

\section{Materials and Methods}

Consecutive patients with acute stroke undergoing endovascular procedures were studied. At patient arrival to the emergency department, a complete evaluation was performed by the neurologist on call, including a complete sonography evaluation (carotid arteries and TCD). ${ }^{1}$ Eligible patients were treated with IV-tPA before the IA procedure. Patients with a persistent arterial occlusion at the end of IVtPA infusion or with contraindications to receive IV-tPA were treated with endovascular procedures. When clinical status allowed, a conscious sedation protocol, avoiding intubation, was preferred. Heparin was administered following femoral artery puncture as a 2000- to 3000-U IV bolus. Only patients with angiographically documented 


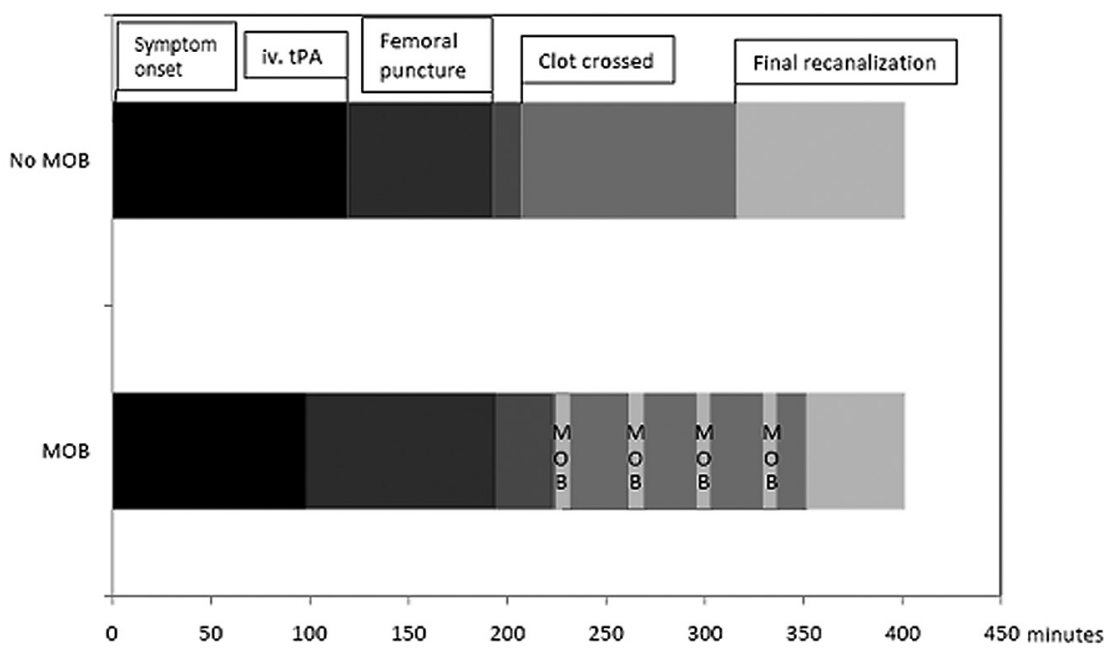

Fig 1. Timing of different procedural steps in patients receiving and not receiving MOB infusion beyond the arterial occlusion. MOB allows intermittently advancing reperfusion for up to 2 hours before final recanalization is achieved.

ICA or MCA occlusion were included in the study. Typically, once the arterial occlusion was angiographically confirmed, a 2.9-2.7F tapered Progreat microcatheter (Terumo, Tokyo, Japan) was advanced through the guiding catheter to the proximal aspect of the clot. Then attempts to cross the thrombus with the microcatheter were made to perform a microcatheter contrast injection to identify the distal aspect of the clot and the patency of the vasculature beyond the occlusion. Interventionalists used repeated local 3- to 5-mg tPA injections (to a maximum of $20 \mathrm{mg}$ ), mechanical clot disruption with the guidewire, and/or the Merci retriever (Concentric Medical, Mountain View, California), according to their preferences and patient characteristics.

The predefined protocol, approved by the local ethics committee, indicates procedure termination when recanalization is achieved or at 6 hours from symptom onset. Timing of all procedural steps was recorded (Fig 1). After time measurements were made in an initial group of patients $(n=40)$, we explored the safety and feasibility of performing repeated injections of oxygenated blood immediately obtained from the femoral artery in a second group of consecutive patients. When the clot could not be crossed with the microcatheter, patients were included in the "No MOB" group $(n=3)$. We finally included 43 patients in the "no MOB injections" group and 17 patients in the MOB group.

In the MOB group, after angiographic confirmation of postocclusion arterial patency, $10-50 \mathrm{~mL}$ of oxygenated blood was manually injected (approximate flow rate, $40 \mathrm{~mL} / \mathrm{min}$ ) through the microcatheter beyond the occlusion every time the clot was crossed during the intervention. Pre- and postocclusion flow was continuously monitored with TCD during the procedure ${ }^{11}$ (PMD100 Digital Transcranial Doppler System; Spencer Technologies, Seattle, Washington), and all changes were recorded.

Recanalization was assessed with the TIMI grading score ${ }^{12}$ at the end of the procedure. For analysis purposes, we considered successful recanalization a TIMI score $\geq 2$. Neurologic status was assessed by a certified neurologist on the patient's arrival, at 12 hours, 24 hours, and at discharge by using the NIHSS. ${ }^{13}$ A 24 -hour CT scan determined the presence of hemorrhagic transformation. We considered hemorrhagic transformation as symptomatic if a neurologic worsening (NIHSS increase, $\geq 4$ points) was accompanied by the presence of blood on follow-up CT. The $\mathrm{mRS}^{14}$ was used to assess clinical outcome at 90 days. The study was approved by local ethics committee.

\section{Statistical Analysis}

Descriptive and frequency statistical analyses were obtained and comparisons were made by using the Statistical Package for the Social Sciences, Version 15.0 (SPSS, Chicago, Illinois). Statistical significance for intergroup differences for categoric variables was assessed by a $\chi^{2}$ test. For continuous variables, the Mann-Whitney $U$ test was used. $P<.05$ was considered statistically significant.

\section{Results}

We studied 60 consecutive patients undergoing urgent endovascular procedures (mean age, $70 \pm 11$ years; median NIHSS score, 20; IR, 18-21). Other baseline variables are shown in the Table. Thirty-three patients (55\%) received IV-tPA before the endovascular procedure. The occluded arteries were the MCA $(63.3 \%$, $n=38)$ and the ICA $(36.6 \%, n=22)$. The mean time from symptom onset to arterial puncture was $193 \pm 77$ minutes. The occluding clot was successfully crossed with the microcatheter in 46 patients $(76.6 \%)$. In these patients, the mean time from femoral artery puncture to microcatheter access to distal vasculature was $25 \pm 21$ minutes. Recanalization could be achieved in 44 patients $(73.2 \%)$ with a mean time from symptom onset of $328 \pm$ 144 minutes. The mean time from first microcatheter clot crossing to final recanalization was $122 \pm 59$ minutes. Repeated manual injections of MOB were performed in 17 patients. Patients with and without MOB presented similar baseline characteristics (Table). The median number of MOB injections was 2 (IR, 2-3), and the mean injected blood volume was $40 \mathrm{~mL}$ (IR, 27.5-50). The mean time from first MOB to final arterial recanalization was $136 \pm 86$ minutes. During MOB, a nonpulsatile flow appeared in previously nonvisible distal branches on TCD display (Fig 2).

One patient receiving $\mathrm{MOB}$ experienced a symptomatic hemorrhagic transformation (5.9\%) compared with $2(4.7 \%)$ among patients not receiving $\mathrm{MOB}(P=.845)$. Recanalization (MOB, $87.5 \%$ versus $67.5 \% ; P=.06)$ was achieved in a similar time from symptom onset (MOB, $350 \pm 49$ versus $316 \pm 177 ; P=.066)$. Median seventh day or discharge NIHSS was similar (MOB: median NIHSS score, 10; IR, $1.5-25$ versus 13.5 ; IR, $2-30 ; P=.657$ ). However when we analyzed the clinical course in those patients in whom re- 


\begin{tabular}{|c|c|c|c|c|}
\hline \multicolumn{5}{|c|}{ Patients characteristics according to treatment group ${ }^{a}$} \\
\hline & $\begin{array}{l}\text { All Patients } \\
(n=60)\end{array}$ & $\begin{array}{l}\text { No MOB } \\
(n=43)\end{array}$ & $\begin{array}{c}\mathrm{MOB} \\
(n=17)\end{array}$ & $\begin{array}{c}P \\
\text { Value }\end{array}$ \\
\hline$\overline{\text { Age }(y r)}$ & $72( \pm 11)$ & $72.1( \pm 10.8)$ & $71.9( \pm 11.2)$ & .960 \\
\hline Female $(\%)$ & 57.1 & 51.2 & 73.3 & .142 \\
\hline Smoking (\%) & 14.9 & 15.6 & 13.3 & .839 \\
\hline Hypertension (\%) & 66.7 & 71.4 & 53.8 & .256 \\
\hline Diabetes $(\%)$ & 18.4 & 19.4 & 15.4 & .748 \\
\hline Atrial fibrillation (\%) & 60 & 58.3 & 64.3 & .703 \\
\hline Admission glycemia (mg/dL) & $128.1 \pm 38.4$ & $125.4 \pm 39.3$ & $135.9 \pm 36.3$ & .465 \\
\hline Systolic blood pressure (mm Hg) & $143.5 \pm 27.5$ & $146.7 \pm 29$ & $134.4 \pm 21.2$ & .205 \\
\hline Diastolic blood pressure $(\mathrm{mm} \mathrm{Hg})$ & $76.5 \pm 15.4$ & $79 \pm 15.7$ & $69.4 \pm 12.4$ & .076 \\
\hline Occluded vessel: MCA/terminal ICA & $63.3 / 36.7$ & $62.8 / 37.2$ & $64.7 / 35.3$ & .823 \\
\hline Baseline NIHSS & $20(18-21)$ & $19(17-21)$ & $21(18-21)$ & .089 \\
\hline IV-tPA (\%) & 55 & 58.1 & 43.8 & .328 \\
\hline Time to IV-tPA (min) & $114 \pm 47.6$ & $119 \pm 52.3$ & $98 \pm 20.9$ & .313 \\
\hline Time to groin & $193 \pm 77$ & $193 \pm 74$ & $192 \pm 73$ & .653 \\
\hline IA-tPA (\%) & 67.3 & 55.3 & 94.1 & .005 \\
\hline IA-tPA dose & $10.6(10.6)$ & $16(9.5)$ & $16.8(4.7)$ & .254 \\
\hline Merci retriever use & 6.6 & 4.3 & 11.7 & .35 \\
\hline Recanalization & 73.2 & 67.5 & 87.5 & .06 \\
\hline Time from symptom to recanalization & $328(144)$ & $316(177)$ & $350(49)$ & .066 \\
\hline Time from groin to recanalization & $125(60)$ & $118(66)$ & $138(47)$ & .310 \\
\hline NIHSS at $12 \mathrm{hr}$ & $18(6.5-20)$ & $18(7-20)$ & $17.5(6-19.2)$ & .79 \\
\hline NIHSS at $24 \mathrm{hr}$ & $16(3-20)$ & $17(2.5-20)$ & $13.5(3-18.5)$ & .544 \\
\hline NIHSS at $48 \mathrm{hr}$ & $14.5(2-20)$ & $14(2-20)$ & $15(2-20)$ & .971 \\
\hline NIHSS at 7 days or discharge & $13(2-30)$ & $13.5(2-30)$ & $10(1.5-25)$ & .657 \\
\hline 24-Hour NIHSS improvement & $4(0-11.5)$ & $3(0-11)$ & $7(2-14)$ & .308 \\
\hline Discharge NIHSS improvement & $9.5(-2-16)$ & $7.5(-9-15)$ & $12(2.7-16.7)$ & .296 \\
\hline Mortality (\%) & 26 & 25.7 & 26.7 & .944 \\
\hline Hemorrhagic transformation (\%) & 34 & 27 & 50 & .089 \\
\hline Symptomatic hemorrhagic transformation (\%) & 5 & 4.7 & 5.9 & .845 \\
\hline $\mathrm{mRS}$ at 3 months & $4(0-6)$ & $3(1-6)$ & $4(0-6)$ & .980 \\
\hline mRS $0-2$ at 3 months (\%) & 40.5 & 40 & 41.6 & .890 \\
\hline
\end{tabular}

Values are expressed as mean $\pm \mathrm{SD}$, median (IR), or percentage.

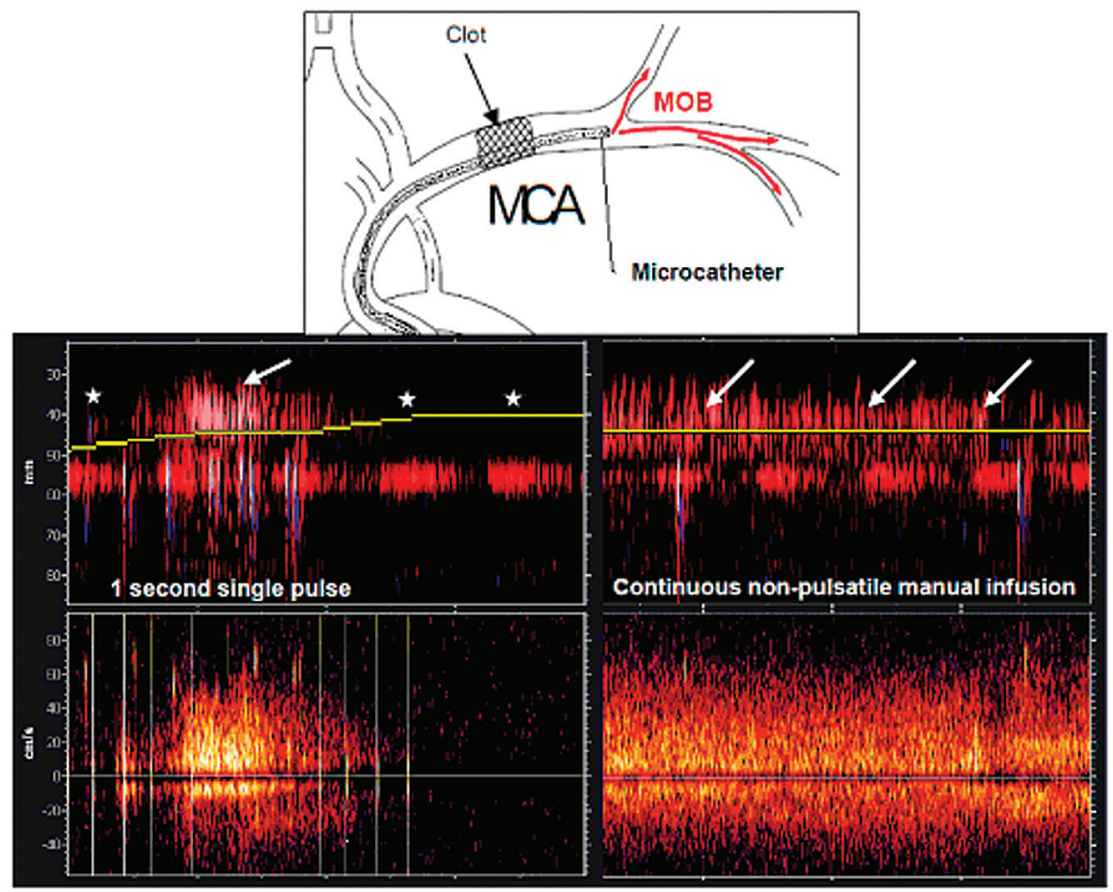

Fig 2. M-mode and TCD recordings during the procedure. Note proximal left MCA occlusion and no detection of flow in the distal MCA branches between a 50- and 30-mm depth (asterisks). During the MOB perfusion beyond the clot, a nonpulsatile flow appears in the distal MCA branches (white arrows). 
canalization was achieved, we observed a trend toward better outcome in patients receiving MOB (median seventh day or discharge NIHSS: (MOB 6 [IR 1-19] versus 14 [IR, 2-30]; $P=.07)$.

\section{Discussion}

This exploratory study shows that microcatheter-mediated perfusion of the ischemic brain with oxygenated blood through the still-occluded artery is feasible and safe. Previous studies showed that in most acute stroke cases, recanalization, unfortunately, many times occurs ${ }^{1,2}$ (spontaneous/later, induced/sooner) too late, leading to large final infarct lesions and disability or death. In the recently published data of the Penumbra Pivotal Stroke Trial, despite the fact that successful recanalization was achieved in $>80 \%$ of patients, only $25 \%$ achieved an mRS $\leq 2$ at 3 months. ${ }^{8}$ The MOB technique theoretically allows transient reperfusion of ischemic penumbra, representing a "fresh gasp" that may "buy time" until accomplishing definitive reperfusion. In our group of patients, $\mathrm{MOB}$ could advance by up to 2 hours the first initial reperfusion compared with patients without MOB, without causing a delay in the time invested to achieve final recanalization.

On the other hand, manually induced blood flow may wash away potentially neurotoxic substances that are locally generated during ischemia or angiographic contrast material that has been reported to increase the rate of symptomatic hemorrhagic transformations. ${ }^{15}$ Because in most cases, IV-tPA was administered, the perfused blood has a high fibrinolytic potential and may favor distal reflow in microvessels or lysis of smaller clots lodged in secondary branches. MOB, moreover, did not increase the rate of symptomatic hemorrhagic transformations, probably because intermittent oxygenated blood perfusion could help maintain the integrity of the blood-brain barrier. If future studies confirm our preliminary data, MOB could be tested to widen the therapeutic time window currently set at 6-8 hours after symptom onset for anterior circulation strokes. The use of continuous TCD monitoring of the flow in the affected vessel confirms the establishment of blood flow in the otherwise stagnant arteries, adding a new indication for the use of TCD during IA procedures. ${ }^{11}$

The idea of establishing catheter systems to reperfuse the brain across the occlusion was described and patented, ${ }^{16}$ but to our knowledge, the technology was never applied beyond the initial prototype stage. Other studies theorized about inducing local hypothermia by perfusing cooling fluids beyond the clot to rapidly achieve the potential benefits of hypothermia, avoiding the systemic complications. ${ }^{17}$ This technique could even overcome the major limitation attributed to systemic administration of neuroprotective drugs. If there is no flow into the ischemic brain, the tested drug will never reach its target, and this may contribute to the repeated deceptions harvested in successive neuroprotection clinical trials. In the future, neuroprotective drugs could be tested by direct local IA administration. Finally, a continuous blood perfusion pump could control and gradually increase the pressure at which blood is injected, avoiding reperfusion injury that seems to be associated with sudden recanalization, abrupt pressure rise, or hyperperfusion. ${ }^{18}$ These systems could be set to continuously perfuse blood through a microcatheter while the neurointerventionalist focuses on recanalizing the artery by working with a second microcatheter. Preclinical animal models are being devel- oped to determine the optimal features of a continuous perfusion system.

These results are preliminary and show only the feasibility and safety of the technique in a small, highly selected patient population. Our study was not designed and powered to determine the possible positive effects of MOB on outcome; however, a preliminary analysis showed an interesting trend toward better outcome in patients receiving $\mathrm{MOB}$ only when recanalization was achieved. This finding supports the hypothesis that MOB is beneficial by "buying time" only if final recanalization is achieved. Prospective studies taking into account baseline variables and total time of ischemia will assess the real clinical benefits of this promising technique.

\section{Conclusions}

In this small series, oxygenated blood delivered through a microcatheter positioned distal to the site of an occlusion was feasible and safe. Its use was associated with a trend toward better outcome. Further study is required to determine its value in the treatment of acute ischemic stroke

\section{References}

1. Molina CA, Montaner J, Abilleira S, et al. Timing of spontaneous recanalization and risk of hemorrhagic transformation in acute cardioembolic stroke. Stroke 2001;32:1079-84

2. Ribo M, Alvarez-Sabin J, Montaner J, et al. Temporal profile of recanalization after intravenous tissue plasminogen activator: selecting patients for rescue reperfusion techniques. Stroke 2006;37:1000-04

3. Arenillas JF, Rovira A, Molina CA, et al. Prediction of early neurological deterioration using diffusion- and perfusion-weighted imaging in hyperacute middle cerebral artery ischemic stroke Stroke 2002;33:2197-203

4. Tissue plasminogen activator for acute ischemic stroke: The National Institute of Neurological Disorders and Stroke rt-PA Stroke Study Group. N Engl J Med 1995;333:1581-87

5. Hacke W, Kaste M, Bluhmki E, et al. Thrombolysis with alteplase 3 to 4.5 hours after acute ischemic stroke. N Engl J Med 2008;359:1317-29

6. IMS II Trial Investigators. The Interventional Management of Stroke (IMS) II study. Stroke 2007;38:2127-35. Epub 2007 May 24

7. Smith WS, Sung G, Saver J, et al. Mechanical thrombectomy for acute ischemic stroke: final results of the Multi MERCI trial. Stroke 2008;39:1205-12. Epub 2008 Feb 28

8. Penumbra Pivotal Stroke Trial Investigators. The Penumbra Pivotal Stroke Trial: safety and effectiveness of a new generation of mechanical devices for clot removal in intracranial large vessel occlusive disease. Stroke 2009;40: 2761-68. Epub 2009 Jul 9

9. Saver JL. Time is brain-quantified. Stroke 2006;37:263-66

10. Ribo M, Molina C, Alvarez B, et al. Buying time for recanalization in acute stroke: arterial blood infusion beyond the occluding clot as a neuroprotective strategy. J Neuroimaging 2009;19:188-90

11. Rubiera M, Cava L, Tsivgoulis G, et al. Diagnostic criteria and yield of real time transcranial Doppler monitoring of intra-arterial reperfusion procedures. Stroke 2010;41:695-9. Epub 2010 Feb 18

12. Chesebro JH, Knatterud G, Roberts R, et al. Thrombolysis in Myocardial Infarction (TIMI) Trial, Phase I: a comparison between intravenous tissue plasminogen activator and intravenous streptokinase - clinical findings through hospital discharge. Circulation 1987;76:142-54

13. Brott TG, Haley EC Jr, Levy DE, et al. Urgent therapy for stroke. Part I. Pilo study of tissue plasminogen activator administered within $\mathbf{9 0}$ minutes. Stroke 1992;23:632-40

14. The Dutch TIA trial: protective effects of low-dose aspirin and atenolol in patients with transient ischemic attacks or nondisabling stroke-TIA Study Group. Stroke 1988;19:512-17

15. Khatri P, Broderick JP, Khoury JC, et al. Microcatheter contrast injections during intra-arterial thrombolysis may increase intracranial hemorrhage risk. Stroke 2008;39:3283-87

16. Lewis BD, Bolduc L. Methods and systems for treating ischemia. US paten 6295990. October 2, 2001

17. Slotboom J, Kiefer C, Brekenfeld C, et al. Locally induced hypothermia for treatment of acute ischaemic stroke: a physical feasibility study. Neuroradiology 2004;46:923-34

18. Pan J, Konstas AA, Bateman B, et al. Reperfusion injury following cerebral ischemia: pathophysiology, MR imaging, and potential therapies. Neuroradiology 2007;49:93-102 